\title{
Rickettsia raoultii and Rickettsia sibirica in ticks from the long-tailed ground squirrel near the China-Kazakhstan border
}

\author{
Shuo Zhao ${ }^{1} \cdot$ Meihua Yang ${ }^{2} \cdot$ Mengmeng Jiang $^{3} \cdot$ Bin Yan $^{1}$. Shanshan Zhao ${ }^{1}$. \\ Wumei Yuan ${ }^{1} \cdot$ Baoju Wang ${ }^{4} \cdot$ Sándor Hornok ${ }^{5} \cdot$ Yuanzhi Wang ${ }^{1}$
}

Received: 28 October 2018 / Accepted: 20 February 2019 / Published online: 25 February 2019

(C) The Author(s) 2019

\begin{abstract}
Spotted fever group (SFG) rickettsiae cause infection in humans, domestic animals and wildlife. To date, no rickettsial agents have been reported in hard ticks from the longtailed ground squirrel (Spermophilus undulatus). A total of 50 adult ticks and 48 nymphs were collected from $S$. undulatus in the border region of northwestern China. Tick species (identified according to morphological and molecular characteristics) included Dermacentor nuttalli, Dermacentor silvarum and Ixodes kaiseri. Based on the cytochrome $c$ oxidase subunit I (COI) haplotype analysis, I. kaiseri from S. undulatus belongs to an ancestral. In addition, all tick samples were analyzed for the presence of rickettsiae by PCR amplification and sequencing of six genetic markers. Rickettsia raoultii and Rickettsia sibirica subsp. sibirica were shown to occur in adults and nymphs of D. nuttalli and D. silvarum. Rickettsia sibirica subsp. sibirica was also detected in an I. kaiseri adult. Dermacentor silvarum and $I$. kaiseri were found for the first time on S. undulatus. Rickettsia raoultii and $R$. sibirica subsp. sibirica were detected in two Dermacentor and one Ixodes species, respectively, suggesting that these rickettsiae circulate in the region of the China-Kazakhstan border by hard ticks infesting $S$. undulatus.
\end{abstract}

Keyword Spermophilus undulatus $\cdot$ Ticks $\cdot$ Rickettsia raoultii $\cdot$ Rickettsia sibirica . Northwestern China

Shuo Zhao, Meihua Yang, and Mengmeng Jiang contributed equally to this work.

Electronic supplementary material The online version of this article (https://doi.org/10.1007/s1049 3-019-00349-5) contains supplementary material, which is available to authorized users.

Yuanzhi Wang

wangyuanzhi621@126.com

Extended author information available on the last page of the article 


\section{Introduction}

The long-tailed ground squirrel (Spermophilus undulatus) has been listed as globally vulnerable by the International Union for Conservation of Nature (IUCN) since 2008 (Ramoslara et al. 2014). This species is mainly distributed in Kazakhstan, Mongolia, Russia and northern China including Heilongjiang, Inner Mongolia and Xinjiang Uygur Autonomous Region (XUAR) (ZipcodeDev Team 2018). According to previous reports, S. undulatus and its ectoparasites are reservoirs of Yersinia pestis, Francisella tularensis and tick-borne encephalitis virus (Zhao et al. 2017; Wang and Yang 1983; Demina et al. 2017). However, only few tick species (i.e., Dermacentor marginatus, Dermacentor nuttalli, Rhipicephalus schulzei and Ixodes persulcatus (Wang and Yang 1983; Demina et al. 2017)) are known to infest $S$. undulatus.

Members of the tick subgenus Pholeoixodes prefer burrowing mammals as hosts (Hornok et al. 2017), thus can be expected to occur on $S$. undulatus. Nevertheless, no previous reports confirmed this. In a study comparing three species of this subgenus (Ixodes kaiseri, Ixodes hexagonus and Ixodes canisuga) the $16 \mathrm{~S}$ rDNA phylogenetic tree reflected that $I$. kaiseri is divided into at least nine COI haplotypes in Europe (Hornok et al. 2017). However, at that time no specimens of I. kaiseri were available from Asia for comparison.

Spotted fever group (SFG) rickettsiae cause infection in humans, domestic animals and wildlife (Maina et al. 2014). At least nine Rickettsia spp. of the SFG had been detected in XUAR (LopezVelez et al. 2015; Guo 2017). According to previous studies, Rickettsia sibirica subsp. sibirica, responsible for Siberian tick typhus, is widely distributed in China, Mongolia, Kazakhstan and Russia, and was also molecularly detected in Spain (Guo 2017; Parola et al. 2013; Byambaa et al. 2008; Palomar et al. 2012). Rickettsia raoultii, the causative agent of tick-borne lymphadenopathy (TIBOLA) or Dermacentor-borne necrosis erythema lymphadenopathy (DEBONEL) (Mediannikov et al. 2008), is prevalent in Mongolia, Europe and the Russian Federation (Oteo and Portillo 2012; Boldbaatar et al. 2017). These examples justify the importance of studying rickettsiae with broad distribution range in Eurasia. Therefore, the aim of this study was to identify ticks infesting S. undulatus, and to molecularly identify Rickettsia species in these ticks.

\section{Materials and methods}

\section{Tick sampling and identification}

A total of 36 S. undulates were captured in Jinghe County (2023 m above sea level; $44^{\circ} 35^{\prime} 59 \mathrm{~N}, 82^{\circ} 53^{\prime} 28 \mathrm{E}$ ), near the wetlands around Ebinur Lake, in the Northwest region of XUAR in July 2017. For this purpose, Sherman traps (H.B. Sherman Traps, Tallahassee, FL, USA) were used, which were placed at the entrances of occupied burrows (TorresPerez et al. 2010). Each survey site included 150 traps that were checked twice a day. Each trap was removed before nightfall and replaced on the survey site the following day.

Ticks were sampled from the entire body of each $S$. undulatus, and were first identified with stereomicroscope (LEICA M165 C) according to standard morphological keys (Hornok et al. 2017). Then the genomic DNA was extracted from all ticks by using the 96 flux automatic nucleic acid extraction instrument with a matching commercial kit (Cell \& Tissue Kit, Bioteke, Beijing, China) according to our previous report (Liu et al. 2018). 
To confirm the morphological identification of tick species, two mitochondrial markers, the $16 S \mathrm{rDNA}$ and the cytochrome $c$ oxidase subunit I (COI) genes (Hornok et al. 2017), were analyzed according to five representative ticks for each tick species. The phylogenic relationships among the representative tick specimens were inferred using MEGA 6.0 software. Twelve nucleotide sequences from our study have been deposited in the GenBank database (16S rDNA: MG656445, MH324406-MH324409; and COI: MH079424, MH279561).

\section{Detection of rickettsial agents and sequence analyses}

Six genetic markers, including 17-kilodalton antigen (17-kDa), surface cell antigen 4 ( $s c a 4$ ), citrate synthase ( $g l t A$ ), cell surface antigen 1 (sca1), and outer membrane proteins $\mathrm{A}$ and $\mathrm{B}(o m p A$ and $o m p B$ ), were amplified from each sample to investigate the presence of SFG rickettsiae (Anstead and Chilton 2013a, b). The primers and PCR cycling conditions in this study are shown in Supplement Appendix Table 1. Each PCR assay included a negative control (distilled water instead of tick DNA template) and a positive control (with DNA from $R$. raoultii obtained from wetlands of Ebinur Lake in XUAR). Purification and sequencing of the PCR products were done as described above (Anstead and Chilton 2013a, b). Phylogenetic trees were constructed using the maximum-likelihood and neighbor-joining methods in MEGA 6.0 software (Guo et al. 2015).

\section{Results}

A total of 98 ticks (50 adult ticks and 48 nymphs) were collected from captured $S$. undulatus. According to morphological characters and the $16 \mathrm{~S}$ rDNA phylogenetic tree, ticks in this study were identified as D. nuttalli, D. silvarum and I. kaiseri (shown in Fig. 1). Based on analysis of COI haplotype, 1-5 nucleotide differences were found in comparison with $I$. kaiseri from Europe. The phylogenetic analysis indicated that I. kaiseri from $S$. undulatus was in an ancestral position to nine European haplotypes ("L to T") (Hornok et al. 2017) (shown in Fig. 2).

Out of 50 adult ticks (26 D. nuttalli, 21 D. silvarum and 3 I. kaiseri) and 48 nymphs (27 D. nuttalli, 21 D. silvarum), 25 adults and 16 nymphs were positive for six Rickettsia genetic markers (17-kDa, gltA, ompA, sca1, sca4 and ompB). Among them, R. sibirica subsp. sibirica was found in nineteen $D$. nuttalli ( 8 nymphs and 11 adult ticks), 13 D. silvarum (6 nymphs and 7 adult ticks) and an adult $I$. kaiseri. In addition, $R$. raoultii was found in five $D$. nuttalli (one nymph and four adult ticks), three $D$. silvarum (one nymph and two adult ticks) (shown in Fig. 3; Table 1). As indicated by five genetic markers (17-kDa, gltA, sca 1 , sca 4 and $o m p B), R$. sibirica subsp. sibirica in this study had sequence similarities in the range of $98.7-100 \%$ when compared to $R$. sibirica from $D$. nuttalli infesting sheep in Jimunai County, XUAR (Guo 2017). In addition, R. raoultii had 99.1-100\% similarity compared to $R$. raoultii strain Khabarovsk (CP010969). The detailed similarities and divergences of the sequences from this study are shown in the Supplement Appendix Table 2. 


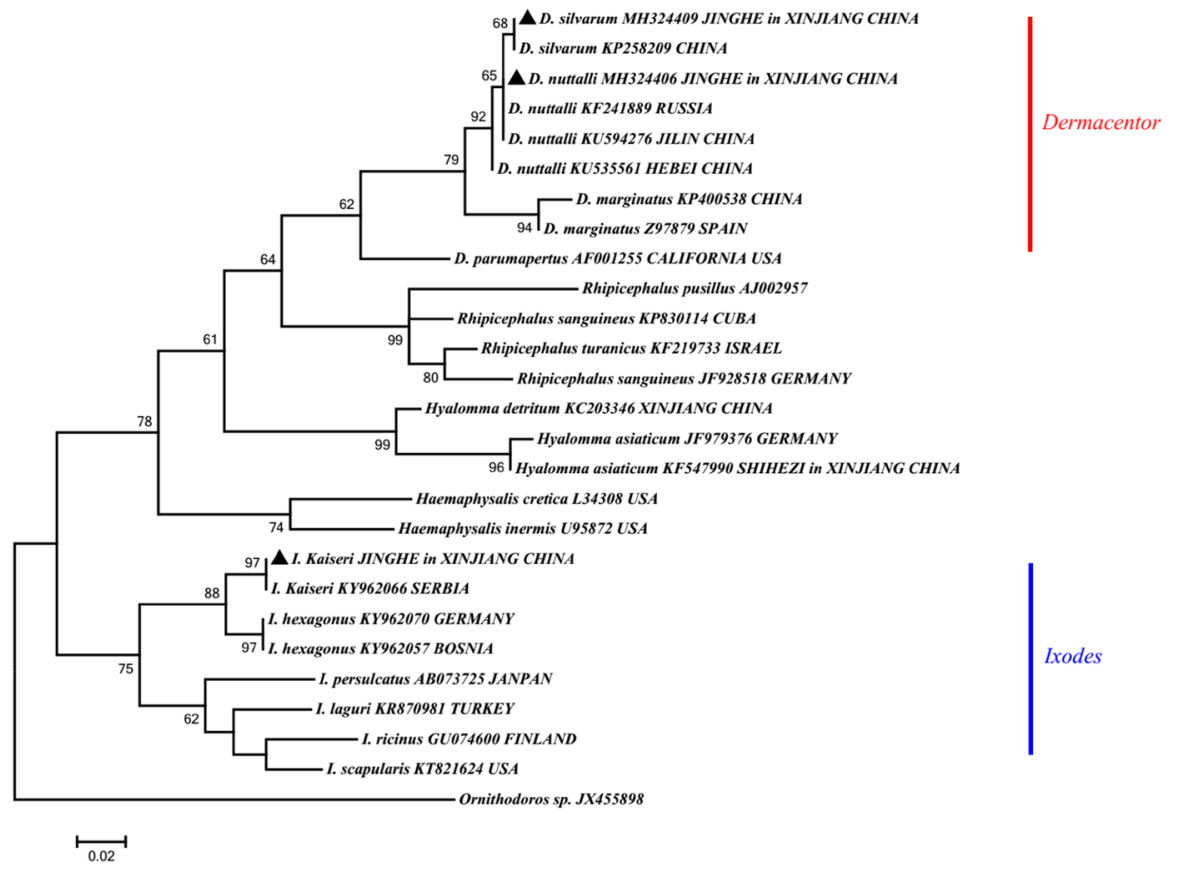

Fig. 1 The phylogenetic tree inferred from the $16 S \mathrm{rDNA}$ sequences of representative tick specimens. The evolutionary history was inferred using the maximum-likelihood method. The new sequences provided by the present study are indicated by a black triangle (containing the accession number). The phylogenetic analyses were conducted using MEGA 6.0 software

\section{Discussion}

Spermophilus undulatus is a burrowing mammalian species, inhabiting mountain areas 1600-3000 m above sea level (a.s.1), with habitats along wetter front hills, forest margins and river valleys (Wang and Yang 1983). Previously, D. marginatus, D. nuttalli, Rh. schulzei and Ixodes persucatus were sampled from S. undulatus (Wang and Yang 1983).

Dermacentor silvarum, a three-host tick species, is widely distributed in North China, Russia and Mongolia (Deng and Jiang 1991; Kulik and Vinokurova 1983). The adult ticks parasitize cattle, horses, sheep, goats, hares and hedgehogs, and the larvae and nymphs mainly infest voles, squirrels and birds (Wang and Yang 1983). On the other hand, I. kaiseri occurs in the western Palearctic, including Germany, Hungary, southern Moldavia, Ukraine, Crimea, Romania, Egypt, Israel, Azerbaijan and Georgia, where it typically infests badgers, foxes, steppe polecats, raccoon dogs, common hedgehogs and domestic dogs (ZipcodeDev Team 2018; Hornok et al. 2017). In the present study, adult ticks (D. nuttalli, D. silvarum and I. kaiseri) and nymphs (D. nuttalli and D. silvarum) were found on S. undulatus in northwestern China. Our results extend the host range of D. silvarum and I. kaiseri. The ancestral phylogenetic position of its COI haplotype from China supports that the genetic diversity of $I$. kaiseri might be related to various geographic locations (Hornok et al. 2017).

To date, $R$. sibirica, including $R$. sibirica subsp. sibirica and $R$. sibirica subsp. mongolotimonae, have been reported from Hyalomma anatolicum, Hyalomma truncatum, Hy. 


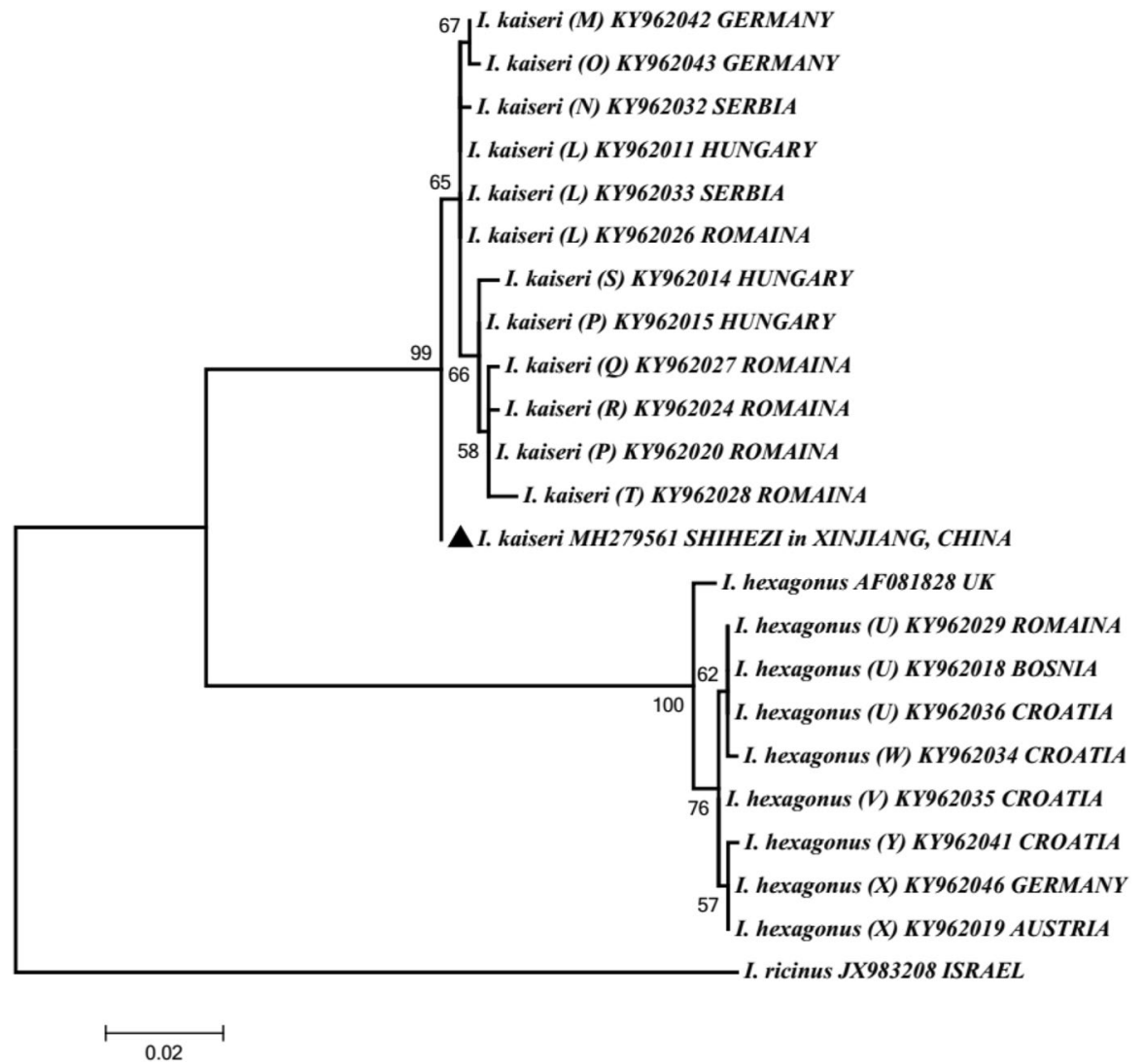

Fig. 2 Phylogenetic tree based on the $C O I$ gene, including sequences obtained in this study and representative sequences of other Ixodes spp.

asiaticum, Rh. pusillus, Dermacentor sinicus, D. nuttalli, D. marginatus, D. reticulatus, D. silvarum, Haemaphysalis yeni, Haemaphysalis concinna and I. persulcatus (Parola et al. 2013). In XUAR, $R$. sibrica subsp. sibirica has already been isolated from $D$. nuttalli in Jinghe County in 1974 (Kong et al. 1982). However, our finding is the first molecular evidence on the presence of $R$. sibirica subsp. sibirica in $I$. kaiseri from $S$. undulatus.

Another Rickettsia species, $R$. raoultii, is highly prevalent in XUAR and its neighboring countries. Among the others, it was detected in Haemaphysalis erinacei from marbled

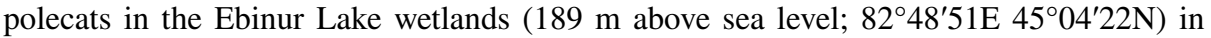
northwest China in 2014 (Anstead and Chilton 2013a, b). Jinghe County, neighboring Ebinur Lake wetlands, has a similar geographic habitat. In the present study, $R$. raoultii was detected in $D$. nuttalli and $D$. silvarum from $S$. undulatus. These findings suggest that $D$. nuttalli, D. silvarum and I. kaiseri parasitizing S. undulatus may serve as reservoirs and carriers for $R$. raoultii and $R$. sibirica.

Jinghe County, with the largest density of $S$. undulatus in China (10-50/hectare), was listed as a main Marmot baibacina-S. undulatus plague focus since 1967 (Wang and Yang 1983). Yersinia pestis was isolated from 3.22\% (66/2051) of S. undulatus (Zhang and Sheng 1991). In our current study, the rickettsial 17-kDa gene was detected from two 


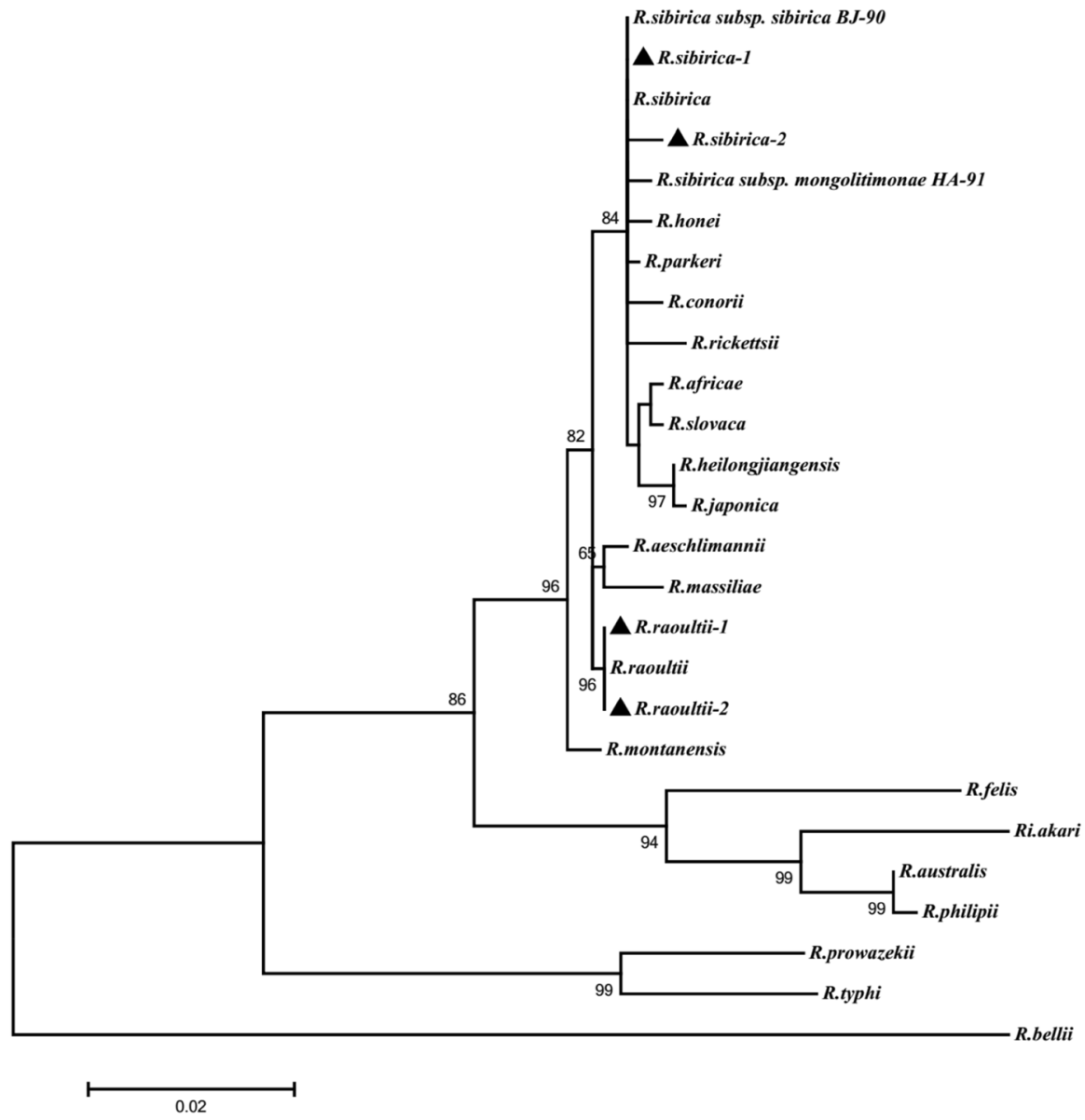

Fig. 3 Phylogenetic tree of the 17-kDa-ompA-gltA-sca4-sca1-ompB concatenated sequence of rickettsial agents in adult ticks and nymphs

Table 1 The prevalence of Rickettsia sibirica and R.raoultii in Dermacentor nuttalli, D. silvarum and Ixodes kaiseri from Spermophilus undulatus

\begin{tabular}{|c|c|c|c|}
\hline Tick species & Rickettsia species & $\begin{array}{l}\text { Prevalence }(\%) \text { in } \\
\text { nymphs }\end{array}$ & Prevalence $(\%)$ in adults \\
\hline \multirow[t]{2}{*}{ D. nuttalli } & R. sibirica & $16.7(8 / 48)$ & $22.0(11 / 50)$ \\
\hline & R. raoultii & $2.1(1 / 48)$ & $8.0(4 / 50)$ \\
\hline \multirow[t]{2}{*}{ D. silvarum } & R. sibirica & $12.5(6 / 48)$ & $14.0(7 / 50)$ \\
\hline & R. raoultii & $2.1(1 / 48)$ & $4.0(2 / 50)$ \\
\hline \multirow[t]{2}{*}{ I. kaiseri } & R. sibirica & 0 & $6.0(3 / 50)$ \\
\hline & R. raoultii & 0 & 0 \\
\hline
\end{tabular}

naturally killed S. undulatus (data not shown). These findings indicate that several vectorborne pathogens co-circulate in Jinghe County, and imply potential risks associated with 
populations of $S$. undulatus. In the future, it is necessary to confirm whether $D$. nuttalli, $D$. silvarum and $I$. kaiseri infesting $S$. undulatus could serve as vectors, transmitting rickettsial agents to $S$. undulatus.

\section{Conclusions}

Dermacentor nuttalli, D. silvarum and I. kaiseri were sampled from S. undulatus near the China-Kazakhstan border, and I. kaiseri in this study had an ancestral COI haplotype when compared to conspecific European sequences. Rickettsia raoultii was detected in the adult and nymph stages of $D$. nuttalli and D. silvarum, and $R$. sibirica also in one $I$. kaiseri adult. These findings extend our knowledge on the range of tick species infesting $S$. undulatus and harboring rickettsial agents.

Acknowledgements The authors are grateful to Dr. Jinliang Sheng (College of Animal Science and Technology) for capturing Spermophilus undulatus.

Author contributions SZ, MY, MJ and YW conceived and designed the study. BY, SZ, WY, BW and WH processed the samples and performed molecular and phylogenetic analyses. SZ and SH contributed to writing the manuscript. All authors read and approved the final version of the manuscript.

Funding This research was supported in part by the National Key Research and Development Program of China (2018ZX10101002-002-007 and 2017ZX10304402-002-005), National Natural Science Foundation of China (81560338 and U1503283), and International Scientific and Technological Cooperation in Bingtuan (2016AH001).

Data Availability The datasets supporting the conclusions of this article are available in GenBank (National Center for Biotechnology Information) [unique persistent identifier and hyperlink to datasets in http://www. ncbi.nlm.nih.gov/genbank/].

\section{Compliance with ethical standards}

Conflict of interest The authors declare that they have no competing interests.

Ethics approval and informed consent This study was approved by the Animal Ethics Committee of Shihezi University (Approval No. AECSU2017-22).

Open Access This article is distributed under the terms of the Creative Commons Attribution 4.0 International License (http://creativecommons.org/licenses/by/4.0/), which permits unrestricted use, distribution, and reproduction in any medium, provided you give appropriate credit to the original author(s) and the source, provide a link to the Creative Commons license, and indicate if changes were made.

\section{References}

Anstead CA, Chilton NB (2013a) A novel Rickettsia species detected in Vole Ticks (Ixodes angustus) from Western Canada. Appl Environ Microbiol 79:7583-7589

Anstead CA, Chilton NB (2013b) Detection of a novel Rickettsia, (Alphaproteobacteria: Rickettsiales) in rotund ticks (Ixodes kingi) from Saskatchewan, Canada. Ticks Tick Borne Dis 4:202-206

Boldbaatar B, Jiang RR, von Fricken ME, Lkhagvatseren S, Nymadawa P, Baigalmaa B, Wang YW, Anderson BD, Jiang JF, Gray GC (2017) Distribution and molecular characteristics of rickettsiae found in ticks across Central Mongolia. Parasit Vectors 10(1):61

Byambaa B (2008) Nature-focal rickettsioses in Mongolia two decades of Russian-Mongolian scientific collaboration. Vestn Ross Akad Med Nauk 7:44-45 
Demina TV, Tkachev SE, Kozlova IV, Doroshchenko EK, Lisak OV, Suntsova OV, Verkhozina MM, Dzhioev YP, Paramonov AI, Tikunov AY, Tikunova NV, Zlobin VI, Ruzek D (2017) Comparative analysis of complete genome sequences of European subtype tick-borne encephalitis virus strains isolated from Ixodes persulcatus, ticks, long-tailed ground squirrel (Spermophilus undulatus), and human blood in the Asian part of Russia. Ticks Tick Borne Dis 8:547-553

Deng GF, Jiang ZJ (1991) Economic insect fauna of China, Fasc 39, Acari: Ixodidae, 1st edn. Science Press, Beijing

Guo LP (2017) Distribution and molecular characteristics of Tick species and tick-borne Rickettsiae, Xinjiang border areas. Shihezi University, Shihezi

Guo LP, Mu LM, Xu J, Jiang SH, Wang AD, Chen CF, Guo G, Zhang WJ, Wang YZ (2015) Rickettsia raoultii in Haemaphysalis erinacei from marbled polecats, China-Kazakhstan border. Parasit Vectors $8: 461$

Hornok S, Sándor AD, Beck R, Farkas R, Beati L, Kontschán J, Takács N, Földvári G, Silaghi C, MeyerKayser E, Hodžić A, Tomanović S, Abdullah S, Wall R, Estrada-Peña A, Duscher GG, Plantard O (2017) Contributions to the phylogeny of Ixodes (Pholeoixodes) canisuga, I. (Ph.) kaiseri, I. (Ph.) hexagonus, and a simple pictorial key for the identification of their females. Parasit Vectors 10:545

Kong SM, Cao GY, Zhang YF, Chen GZ (1982) Isolation and identification of Rickettsia from tick-borne spotted fever group in Jinghe County of Xinjiang. Mic Bull 9:13-15

Kulik IL, Vinokurova NS (1983) Range of the tick Dermacentor silvarum in the USSR. Med Parazitol (Mosk) 52:23-28

Liu XF, Yan B, Wang Q, Jiang MM, Tu CC, Chen CF, Hornok S, Wang Y (2018) Babesia vesperuginis in Common Pipistrelle (Pipistrellus pipistrellus) and the Bat Soft Tick Argas vespertilionis in the People's Republic of China. J Wildl Dis 54:419-421

LopezVelez R, Palomar AM, Oteo JA, Norman FF, Pérez-Molina JA, Portillo A (2015) Novel Candidatus rickettsia species detected in nostril tick from human, Gabon, 2014. Emerg Infect Dis 21:325-327

Maina AN, Jiang J, Omulo SA, Cutler SJ, Ade F, Ogola E, Feikin DR, Njenga MK, Cleaveland S, Mpoke S, Ng'ang'a Z, Breiman RF, Knobel DL, Richards AL (2014) High prevalence of Rickettsia africae variants in Amblyomma variegatum ticks from domestic mammals in rural western Kenya: implications for human health. Vector Borne Zoonotic Dis 14:693-702

Mediannikov O, Matsumoto K, Samoylenko I, Drancourt M, Roux V, Rydkina E, Davoust B, Tarasevich I, Brouqui P, Fournier PE (2008) Rickettsia raoultii sp. nov. a spotted fever group rickettsia associated with Dermacentor ticks in Europe and Russia. Int J Syst Evol Microbiol 58:1635-1639

Oteo JA, Portillo A (2012) Tick-borne rickettsioses in Europe. Ticks Tick Borne Dis 3:271-278

Palomar AM, Santibañez P, Mazuelas D, Roncero L, Santibañez S, Portillo A, Oteo JA (2012) Role of birds in dispersal of etiologic agents of tick-borne zoonoses, Spain, 2009. Emerg Infect Dis 18:1188-1191

Parola P, Paddock CD, Socolovschi C, Labruna MB, Mediannikov O, Kernif T, Abdad MY, Stenos J, Bitam I, Fournier PE, Raoult D (2013) Update on tick-borne rickettsioses around the world: a geographic approach. Clin Microbiol Rev 26:657-702

Ramoslara N, Koprowski JL, Kryštufek B, Hoffmann IE (2014) Spermophilus citellus (Rodentia: Sciuridae). Mamm Species 913:71-87

Torres-Perez F, Wilson L, Collinge SK, Harmon H, Ray C, Medina RA, Hjelle B (2010) Sin Nombre virus infection in field workers, Colorado, USA. Emerg Infect Dis 16:308-310

Wang SB, Yang GY (1983) Rodent fauna of Xinjiang, 1edn Xinjiang People's publishing house, Urumqi

Zhang HQ, Sheng GJ (1991) Xinjiang long tail mouse The plague overview about Spermophilus undulatus in Xinjiang Uygur Autonomous Region. Endem Dis Bull 5:114-117

Zhao SS, Pulati Y, Yin XP, Li W, Wang BJ, Yang K, Chen CF, Wang YZ (2017) Wildlife plague surveillance near the China-Kazakhstan border: 2012-2015. Transbound Emerg Dis 64(6):48-51

Zipcodezoo. ZipcodeDev Team (2018). http://zipcodezoo.com/

Publisher's Note Springer Nature remains neutral with regard to jurisdictional claims in published maps and institutional affiliations. 


\title{
Affiliations
}

\author{
Shuo Zhao ${ }^{1} \cdot$ Meihua Yang $^{2} \cdot$ Mengmeng Jiang $^{3} \cdot$ Bin Yan $^{1}$. Shanshan Zhao ${ }^{1}$. \\ Wumei Yuan ${ }^{1} \cdot$ Baoju Wang ${ }^{4} \cdot$ Sándor Hornok ${ }^{5}$. Yuanzhi Wang ${ }^{1}$ \\ Shuo Zhao \\ 954855721@qq.com \\ Meihua Yang \\ 1328251980@qq.com \\ Mengmeng Jiang \\ 577674273@qq.com \\ Bin Yan \\ 1262321593@qq.com \\ Shanshan Zhao \\ 1393819696@qq.com \\ Wumei Yuan \\ 348781741@qq.com \\ Baoju Wang \\ bjwang73@163.com \\ Sándor Hornok \\ hornok.sandor@univet.hu \\ 1 Department of Basic Medicine, School of Medicine, Shihezi University, Shihezi, Xinjiang, China \\ 2 Department of Forestry, College of Agriculture, Shihezi University, Shihezi, Xinjiang, China \\ 3 Department of Veterinary Medicine, College of Animal and Science, Shihezi University, Shihezi, \\ Xinjiang, China \\ 4 Department of Infectious Diseases, Union Hospital of Tongji Medical College, Huazhong \\ University of Science and Technology, Wuhan, China \\ 5 Department of Parasitology and Zoology, University of Veterinary Medicine, Budapest, Hungary
}

